\title{
Analysis and Representation of QoS Attributes in Cloud Service Selection
}

\author{
Mona Eisa, Muhammad Younas, Kashinath Basu \\ School of Engineering, Computing and Maths \\ Oxford Brookes University \\ Oxford, United Kingdom \\ \{mona.eisa-2015, m.younas, kbasu\}@brookes.ac.uk
}

\begin{abstract}
Cloud service selection is complicated by the prospect that there exist a large number of services and each service is characterized by multiple QoS attributes. Various commercial tools have been developed in order to help cloud consumers with selecting best cloud services. This paper provides an in-depth analysis of the three commercial cloud service selection tools as well as the way they represent QoS attributes. Accordingly, it proposes a new model that succinctly represents QoS attributes which cloud consumers can easily use (and understand) when selecting cloud services. The proposed model also classifies the QoS attributes into four main categories of technical, strategic \& organizational, economic and political \& legislative. These QoS attributes can also be seamlessly fed into the multi-criteria decision (e.g., MCDM) -- which compares and ranks different QoS attributes of multiple alternatives in order to decide which services are most suitable for cloud consumers.
\end{abstract}

Keywords - cloud, service selection, quality of service, QoS attributes

\section{INTRODUCTION}

Cloud computing provides on-demand computing resources such as compute power, storage, servers, and software applications and software development environment [1]. The use and growth of cloud computing has exponentially increased [2]. More and more cloud providers are entering into the cloud business market and are offering various types of cloud services. Realising the benefits of cloud (e.g., reduced IT expenditure, on-demand services, large pool of resources), increasing number of businesses and organizations want to outsource their (in-house) IT services to the cloud. However, outsourcing IT services to the cloud, careful decisions are to be made in the selection of cloud services. This is because, cloud services are provided with varying level of quality of service (QoS) such as security, reliability, performance, and usability, to name a few.

Given the large number of cloud providers and cloud services, the challenge for cloud service consumers is to select those services that meet their QoS requirements and the terms and conditions of SLA (Service Level Agreement). Cloud service selection is a process used to select appropriate cloud services. It is a complicated process as there exists a large number of services and each service is characterized by multiple QoS attributes such as efficiency, security, reliability and so on. Various commercial tools have been developed in order to help cloud consumers with selecting best cloud services.

This paper investigates into the analysis and representation of QoS requirements. It studies three widely available commercial tools developed for cloud service selection and filtering. These include Intel Cloud Finder, RankCloudz and Cloudorado. The objectives of the analysis of existing tools are to guide our study in (1) finding issues related to the current tools (2) identifying the most important QoS factors affecting the selection of cloud services. Accordingly, it proposes a new model which contributes to the cloud service selection in multiple ways. First, the proposed model succinctly represents QoS attributes which cloud consumers can easily use (and understand) when selecting cloud services. Second, based on the classification framework, Janssen and Joha (2011), the proposed model classifies QoS attributes into four main categories of technical, strategic \& organizational, economic and political \& legislative. These QoS attributes can also be seamlessly fed into the multi-criteria decision (e.g., MCDM) -which compares and ranks different QoS attributes of multiple alternatives based on cloud consumer's selection of attributes and their relative weightage in order to decide which services are most suitable for cloud consumers

The rest of the paper is organized as follows. Section II describes the related work. Section III explains cloud service selection. The analysis of current cloud service selection tools is explained in Section IV. In Section V. the proposed model for representing and structuring the QoS attributes is presented.

\section{RELATED WORK}

Cloud service selection is an area that has been the subject for many research works for the past several years. There are many aspects in cloud service selection. One of the important aspects that is closely related to cloud service selection is Quality of Service (QoS). QoS attributes are used in cloud selection criteria. Some studies use single quality attribute while some other studies use a multi-dimensional QoS attributes. Salama et al. [3] propose a cloud service selection based on a group of broad, multi-dimensional QoS attributes. An integrated QoS-assured utility model is proposed to address 
the problem of cloud service selection. There are two phases of selection where users need to specify specific scores for the QoS attributes as the requirement to select cloud services.

While some researches limit the selection process within a set of pre-defined cloud service criteria, other researches take advantage of recent efforts that formulate a set standardized cloud QoS attributes called SMI (Service Measurement Index) [4]. Garg et al. [5], [6] create SMICloud which is a framework for comparing and ranking cloud services. SMICloud uses standardized SMI attributes [4] in order to compare cloud providers based on user requirements. Baranwal et al. [7] propose a framework for selecting best cloud service providers using SMI framework as the standard QoS attributes. The difference with SMICloud is that this framework utilizes ranked voting method to find the best cloud services. A list of metrics of efficient cloud providers acts as the voter and the cloud providers act as the candidates. In this methodology voter ranks the candidates in order of preference.

The aforementioned studies related to QoS attributes in cloud service selection come from a more technical point of view. In addition, cloud computing and service selection can also be seen as a business decision for many organizations. Janssen et al. [8] analyze the challenge to adopt cloud computing, particularly Software as a Service (SaaS) in the public sector. Analysis in this study looks at the advantages, disadvantages and challenges in implementing SaaS from technical and non-technical aspects. Janssen et al. define four categories for SaaS, namely, strategic and organizational, political and legislative, technical, and economic. The decision to use this categorization is based on a previous study by Baldwin et al. [9]which is related to the IT outsourcing in banking sector. In their study, Baldwin et al., propose a set of motives for outsourcing decision where these motives are categorized into four categories as in Janssen et al. [8].

\section{Cloud Service SElection}

Cloud service providers (or vendors) are companies that offer different services such as data storage, software applications, networks, memory, and compute services in the cloud. Cloud services are offered using service provisioning models such as SaaS (Software-as-a-Service), PaaS (Platformas-a-Service, and IaaS (Infrastructure-as-a-Service).

Given the large number of cloud providers and cloud services, the challenge for cloud service consumers is to select those services that meet the required QoS criteria.

According to the International Organization for Standardization in ISO 9000, quality is "the totality of features and characteristics of a product or service that bear on its ability to satisfy stated or implied needs" [10]. In ISO's latest update standard, namely ISO 9000:2015, however, quality is defined as "a degree to which a set of inherent characteristics of an object fulfills requirements" [11]. Based on these definitions the quality of a cloud service can be defined as a degree to which a set of attributes of a service fulfills stated requirements.
This paper investigates into the analysis, representation and classification of the QoS attributes of cloud services. It first studies and analyses three widely available commercial tools which have been developed for cloud service searching, filtering, and selection. These include, Intel Cloud Finder, RankCloudz and Cloudorado.

\section{Analysis of Cloud SERVICE SELECtion ToOlS}

This section presents an analysis of three commercial tools. The objectives of the analysis are to guide our study in; finding issues related to the current tools; and identifying the most important QoS factors affecting the selection of cloud services.

\section{A. Intel Cloud Finder}

There are two types of searches in Intel Cloud Finder, namely Quick Search and Detailed Search where each type of search presents a different sets of cloud QoS attributes [12]. In Quick Search users can quickly search for a cloud services that satisfy their requirements by choosing features that closely match their specification. There are five top-level attributes in Quick Search: Interface Model, Development Support, Subscription Options, Geography and Verticals. Each of these five attributes have several options from which users can choose the ones that are close to their requirements.

- Interface Model reflects the type of interaction available for users for accessing and configuring their Cloud infrastructure that cloud providers are providing.

- Development Support reflects the added support that allows users to customize their platform such as storage services as well as custom OS images.

- Subscription Options are the available ways for users to purchase or pay for the services and infrastructure they are leasing.

- Geography is for the region where a cloud provider is operating.

- Verticals reflect the industry where the user would use the services and infrastructure they are trying to acquire.

Quick search is suitable for novice users because of the quick and simple process as well as the relatively simple presentation of the QoS attributes. However, explanation is lacking in quick search. Out of five attributes and a total of twenty-five options, Intel only provides explanation for three options which are Pay-as-You-Go (under Subscription Option), Spot Instance Bidding (under Subscription Option), and Reserved Instances (under Subscription Option). The complete QoS attributes for Intel Cloud Finder quick search is represented in Fig. 1. 


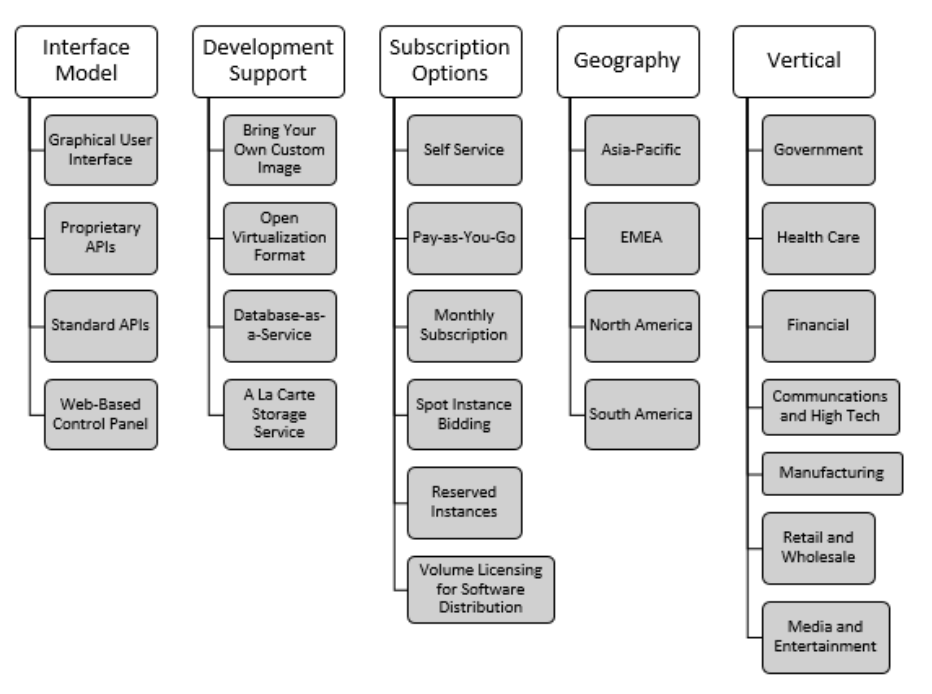

Figure 1 Intel Cloud Finder - Quick Search QoS Attributes

The Detailed Search allows users to have more flexibility and lets users give importance to the attributes presented to them. Similar to the Quick Search, the attributes were categorised in a way that contain sub-attributes, and each sub-attribute has several options; all of which contain brief description of what the features are. The parameters are Security, Usability, Quality, Availability, Technology and Business.

- Security reflects the ways users can access as well as protect the infrastructure they are leasing. It also implies different regulations and standards.

- Usability reflects the service capabilities and monitoring features that are available to the users when accessing and leasing an infrastructure.

- Quality is composed of how easy it is for the users to use the services as well as level of agreement available for user to choose.

- Availability reflects the way for users to access and control their infrastructure in cases of emergencies. It also implies location availability where a particular cloud provider operates in a particular region.

- Technology reflects functional requirements for cloud service where hardware and software that enable Cloud Computing is presented to the user as different features.

- Business gives definition of the cloud providers detailing information about the cloud providers such as revenue, data centers locations, etc.

The 2-level QoS attributes for Intel Cloud Finder Detailed search is represented in Fig. 2.
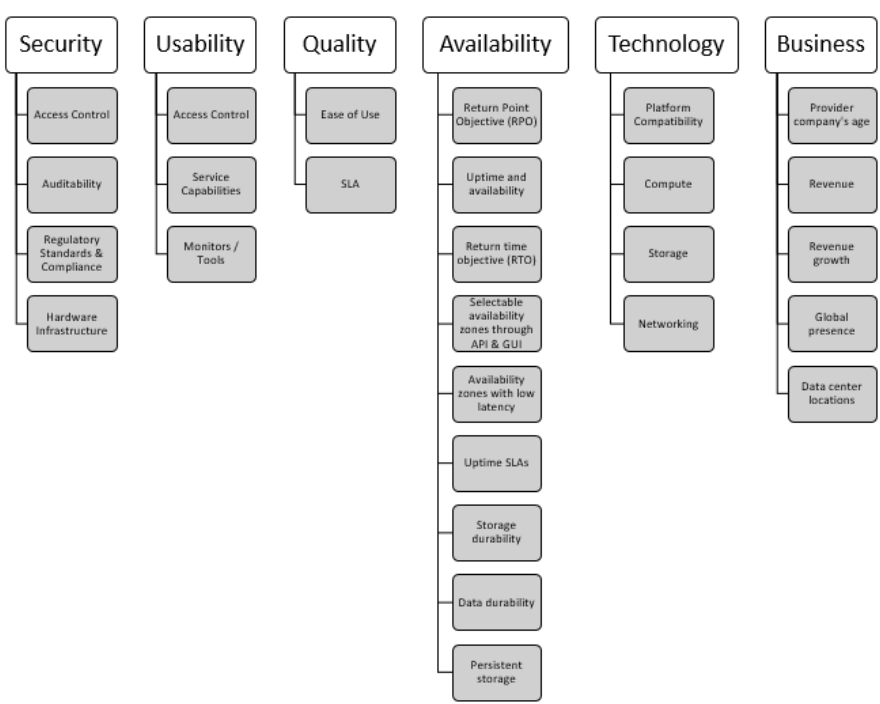

Figure 2 Intel Cloud Finder - Detailed Search QoS Attributes

Users need to determine the level of importance of each option under each sub-parameter. There are four different levels of importance, ranging from the 'most importance' to the 'least importance': essential, desirable, future, N/A. The fourth option is the default value for all options. Intel provides brief definition of all the features in the Detailed Search. Although the explanation for all attributes are fairly covered, users are presented with an overwhelmingly large number of features and options. In the selection process users need to review all of these options in order to narrow down the cloud provider candidates.

\section{B. RankCloudz}

RankCloudz selection process requires users to determine the level of importance of a set of attributes. There are five different cloud services that user can choose: Dev \& Test Infrastructure, Virtual Data Centre, Enterprise Apps \& Hosting, Storage \& Backup and Big Data \& Analytics. Each of service has its own set of attributes, ranging between 11 to 14 attributes [13]. Fig. 3 below depicts the attributes underneath each of the five RankCloudz cloud services.

The level of importance in RankCloudz is represented by a scale that starts from 0 as the lowest importance and it goes up to 10 as the highest importance. RankCloudz ranks the cloud providers depending on the attributes and the priority that user has set for each attribute. Though the process of assigning score that reflects the level of importance is quite straightforward, RankCloudz does not provide explanation for each of these attributes. Novice user might find the process difficult because of the lack of attributes' description. 


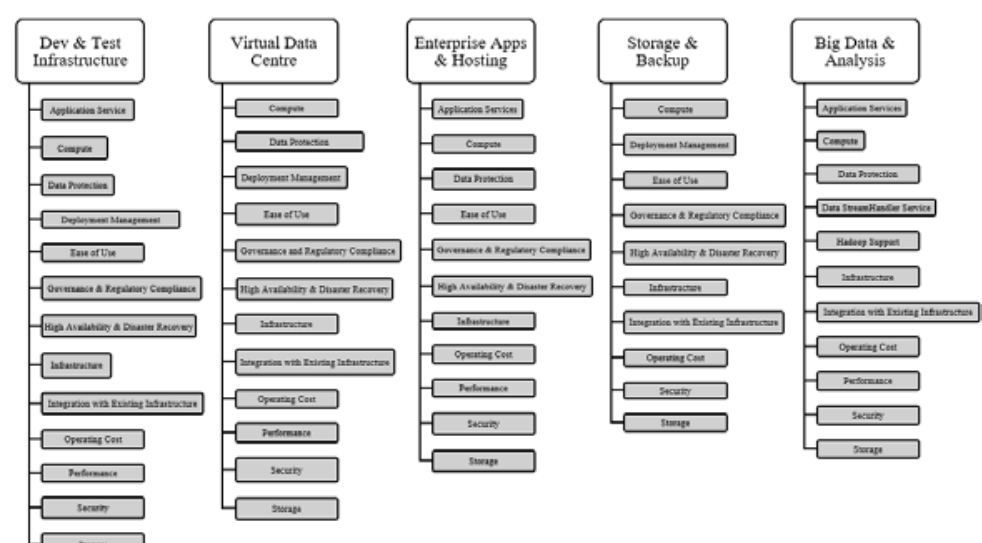

Figure 3 RankCloudz QoS Attributes

\section{Cloudorado}

Cloudorado selection process works like Intel Cloud Finder Quick Search where user chooses an option from each attribute that matches their requirements [14]. Cloudorado differentiates three different types of cloud services: Cloud Server, Cloud Hosting and Cloud Storage. Each of these cloud services has different set of attributes. Cloud Server and Cloud Hosting categories have the same non-functional attributes presented to the users but with different functional attributes. Users can configure both the functional and non-functional attributes that satisfy their requirements. Cloudorado presents user with a lot of detailed attributes, but it also provides brief description for all of these attributes. The selection and QoS attributes hierarchy is represented in Fig. 4.

\section{Analysis of QoS Attributes in Existing Service Selection Tools}

The comparison of QoS attributes in existing tools is done with the consideration that this is related to cloud service selection. In the process of cloud selection QoS attributes play an important part as the defining characteristics that help users match their own requirement with that of cloud services. User is an important entity in this analysis because the end goal is to provide QoS attributes that are structured in such a way that make the cloud service selection process easier for users, both novice users and expert users. With this in mind, we use the following criteria to analyze and compare QoS attributes from the three existing tools as described above.

- Structure: When broken down to the lowest, mostdetailed level, the high number of cloud QoS attributes can be overwhelming. Because of the large number of attributes, it is important to organize them in such a way that is easy to use and understand by users. Structure is an important factor because structure provides a frame to organize these many attributes. Structure represents the ability to place and arrange attributes into something that is less complex, or simple enough to understand. In this case, we consider two types: hierarchical and non-hierarchical structures. All of the previous studies related to QoS attributes attempt to create some sort of hierarchy for QoS attributes.

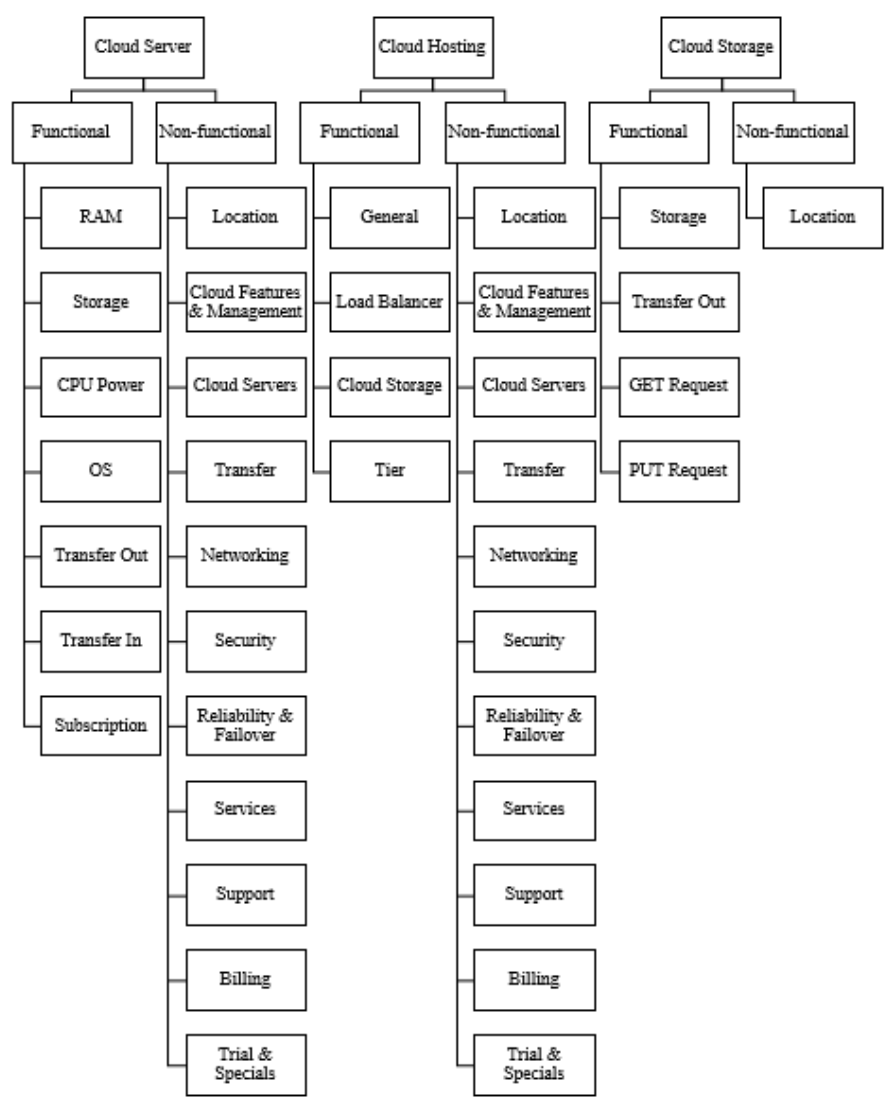

Figure 4 Cloudorado QoS Attributes

- Number of Levels: Related to the previous criteria, the number of levels indicates how many levels there are in the QoS attributes hierarchy. Number of levels in a QoS attributes hierarchy can indicate how straightforward a cloud service selection process can be. Having more levels may result in complex service selection process which may not be easy to use by novice users.

- Flexibility in Hierarchy: The flexibility in hierarchy indicates the ability for the hierarchy structure to present the QoS attributes to the user in different depth. This criteria is considered with the idea that cloud service selection users have different level of knowledge in cloud computing. With flexibility, the same hierarchy can be presented to the user in different depths. For instance, one user might choose to see the QoS attributes presented in a simpler, twolevel hierarchy, while another user who is more expert might choose to see the QoS attributes presented in a more detailed, three-level hierarchy. With the ability to present the same structure in different depths, a cloud selection tool will be able to cater to user from all levels of cloud computing knowledge.

- Type of User Input: Cloud selection process is done by getting input from the user where user essentially tells the system what kind of requirements they want from the prospective cloud provider. Different 
selection tools have different ways to get input from the user.

- Description of QoS Attributes: Description for each QoS attribute is needed to provide the user brief explanation of the attributes. In addition, QoS attribute description can also avoid confusion or misunderstanding over the meaning of certain attributes. The availability of QoS attributes description will help non-technical and novice user.

Table 1 provides a comparison of the QoS attributes of Intel Cloud Finder (Quick Search and Detailed Search), RankCloudz and Cloudorado.

Intel quick search presents users with five categories of attributes. Each of them have options which users can directly choose. There is only one level of QoS attributes categorization. This also means there is no option to go into a more detailed level. The process is very simple and straightforward, but the number of attributes involved is limited. Intel Quick Search provides attributes definition only partially and not all attributes presented have explanation.

Intel Detailed Search provides a more detailed type of cloud selection process. In it, attributes are represented in a 2level hierarchy. However, there is no flexibility in the hierarchy in that user can't choose to give input for top-level attributes only. Underneath each of level 2 attributes are options for which user needs to determine the level of importance: essential, desirable, future, N/A. While there is a large number of options presented to the user, Intel Detailed Search provides brief description for all attributes.

RankCloudz lumps technical and business requirements together in one non-hierarchical structure. Because of the flat structure of the attributes, there is no flexibility in hierarchy. The number of attributes involved is fairly manageable where user only needs to give input for 11 to 14 attributes. For each of these attributes user only needs to determine the importance level which ranges from 0 to 10 . Despite the seemingly straightforward process, RankCloudz doesn't provide description for any of the attributes.

Cloudorado presents attributes in a subtle hierarchy structure. Subtle hierarchy means that the hierarchy is not explicitly written but it is apparent in the user interface where Cloudorado displays functional attributes as one group and the non-functional attributes as another group. For the nonfunctional attributes group, Cloudorado divides these into a more detailed category resulting in a 2-level hierarchy for the non-functional attributes. User doesn't have any flexibility to choose which level of the hierarchy they choose to give input to. There is quite a large number of attributes which are presented to the user. However, user doesn't have to select these options as Cloudorado has set a default value for some of the options. Cloudorado also provides brief description for all QoS attributes.
TABLE 1 SUMMARY OF QOS ATTRIBUTES COMPARISON

\begin{tabular}{|l|l|l|l|l|}
\hline \multirow{2}{*}{ Criteria } & \multicolumn{2}{|l|}{ Intel Cloud Finder } & RankCloudz & Cloudorado \\
\cline { 2 - 3 } & Quick & Detailed & & \\
\hline Structure & $\begin{array}{l}\text { Non- } \\
\text { hierarchy }\end{array}$ & Hierarchy & Non-hierarchy & Hierarchy \\
\hline No. of Level & 1 & 2 & 1 & 2 \\
\hline $\begin{array}{l}\text { Flexibility in } \\
\text { Hierarchy }\end{array}$ & None & None & None & None \\
\hline $\begin{array}{l}\text { Type of User } \\
\text { Input }\end{array}$ & $\begin{array}{l}\text { Option } \\
\text { selection }\end{array}$ & $\begin{array}{l}\text { Level of } \\
\text { importance }\end{array}$ & $\begin{array}{l}\text { Level of } \\
\text { importance }\end{array}$ & $\begin{array}{l}\text { Option } \\
\text { selection }\end{array}$ \\
\hline $\begin{array}{l}\text { Description } \\
\text { of QoS } \\
\text { Attributes }\end{array}$ & Partial & Available & None & Available \\
\hline
\end{tabular}

\section{THE Proposed MOdEL FOR QOS}

The proposed model follows the following phases in order to structure and represent the QoS attributes of cloud services.

\section{A. Collection Phase}

This phase is to collect relevant QoS attributes of cloud services. In the proposed model, QoS attributes are gathered from different type of sources, namely, existing cloud service selection tools, cloud service providers, previous researches related to QoS attributes, and existing standardized service QoS.

Existing cloud service selection tools are included because one of the main objectives of proposed model is to improve existing selection tool by simplifying the selection process. This objective is obtained by referencing several existing selection tools and building upon what currently exists in order to create a better selection framework. For this reason, QoS attributes in existing selection tools are reviewed and included in this QoS attributes classification process.

The second source is cloud service providers. The proposed model looks into several cloud service providers and gathers the QoS attributes from the information published on providers' websites. Therefore, QoS attributes publicly listed on cloud providers' website are included in this process.

The third source is previously published research work. The proposed model takes into account some of the QoS attributes which are commonly used in the research work related to cloud service QoS.

The final source is existing standardized service QoS. The current standardized framework on service attribute is Service Measurement Index (SMI) which is developed by the Cloud Service Measurement Initiative Consortium (CSMIC) [4]. Although the proposed model does not fully adopt the SMI framework, QoS attributes are considered and included in the QoS classification process. 


\section{B. Categorisation Phase}

As described above, cloud service QoS include many attributes that are related to the characteristics and the delivery of cloud service. QoS attributes can be related to the technical aspects of cloud services as well as business aspects of cloud services. Because of the seemingly endless cloud QoS attributes, categorizing them in a structured way is important as it will help in sorting out these attributes. In order to cater for technical as well as business aspects of cloud services, the proposed model represents QoS attributes into four categories: Technical, Strategic \& Organizational, Economic, and Political \& Legislative. This categorization scheme is adopted from Baldwin et al. [9] and also Janssen et al. [8]. However, the focus of the work presented in [8] and [9] is different than the proposed model.

For instance, Baldwin et al., look at the prospect of outsourcing banking information system while in the case of Janssen et al. they look at the prospect of adopting cloud computing technology, specifically SaaS technology. But the similarity of the proposed model to the models presented in [8] and [9] is the 'technology adoption by an organization'. The main objective of cloud service selection is to help businesses and organizations in selecting cloud services and thus adopting cloud technology. The proposed model therefore adopted the aforementioned four-category categorization scheme.

Further, it is paramount to include business-related consideration into the service selection process. Cloud computing technology consists of not only technical aspect, but also business aspects. In the proposed model, it is considered as important that QoS attributes should cover both technical and non-technical.

Furthermore, from the review of several sources regarding cloud QoS attributes discussed in the previous Collection Phase above, it is concluded that different providers, different tools, different studies or researches use different ways to structure or categorize QoS attributes. Selecting a categorization scheme, that covers a broader and more general scope, categorizes cloud service QoS attributes into four top categories [8][9] : technical, strategic \& organizational, economic, and political \& legislative. Each of these four categories will be represented by at least one attribute in the proposed model. This is to make sure the attributes in the proposed model cover all aspects of cloud services. Each of the four categories is briefly described as follows [15]:

- Technical - factors related to the capabilities and limitations of the technology

- $\quad$ Strategic \& Organizational - factors related to an enterprise's organizational and strategic goal

- Economic: factors related to the financial aspects of cloud services

- $\quad$ Political \& Legislative: compliance with standards

In the proposed model, the distribution of the first-level QoS attributes according to the four categories can be seen in the diagram below.

\begin{tabular}{|l|}
\hline Technical \\
-Security \\
-Usability \\
- Assurance \\
-Performance \\
Strategic \& Organization \\
-Company Performance \\
Economic \\
-Pricing \\
Political \& Legislative \\
-Compliance
\end{tabular}

Figure 5 Categorization Of First Level Attributes

\section{Structuring Phase}

This phase represents the QoS attributes in a hierarchical way because of two reasons. The first one is because hierarchy structure adhere to the AHP (Analytic Hierarchy Process) methodology that is commonly used in the cloud service selection framework. AHP methodology breaks down the problem in several levels and models the problem in a hierarchical structure. The second reason is that hierarchical structure provides flexibility to users. This is especially tied in with the goal of the proposed model that takes into account user's knowledge of cloud computing. By having hierarchical structure, it is easy to place a more general attributes at the top level and the more detailed attributes at the lower levels. And in so doing, the proposed model has the ability to present novice users with top-level, general QoS attributes as well as to present expert users with the more detailed attributes situated at the lower level. This kind of flexibility is not provided in the three existing selection tools reviewed in the previous section of this paper.

In the proposed model there are seven top-level attributes: security, usability, assurance, performance, company performance, pricing, compliance. The 2-level structure of the QoS attributes in the proposed model is graphically represented in Fig. 6, while the complete attributes can be found in Figures 7,8 and 9 .
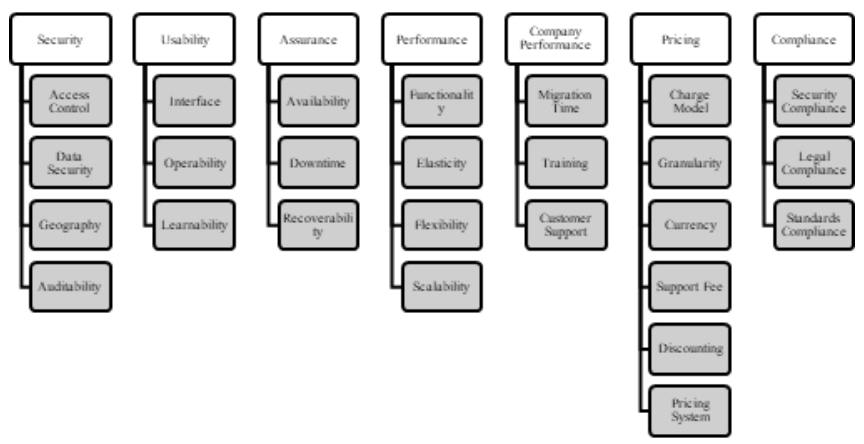

Figure 6 QOS Attributes Hierarchical Structure 

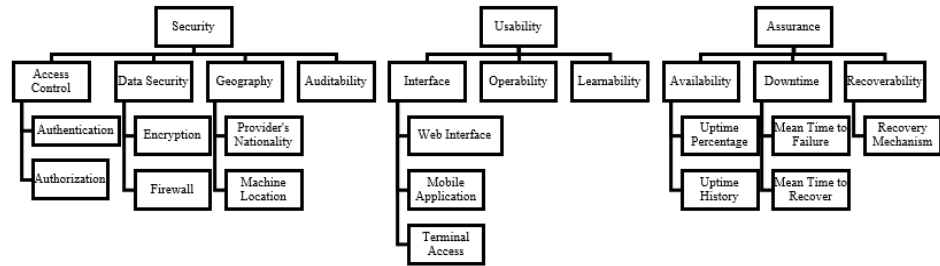

Figure 7 QoS Attributes for Security, Usability, and Assurance

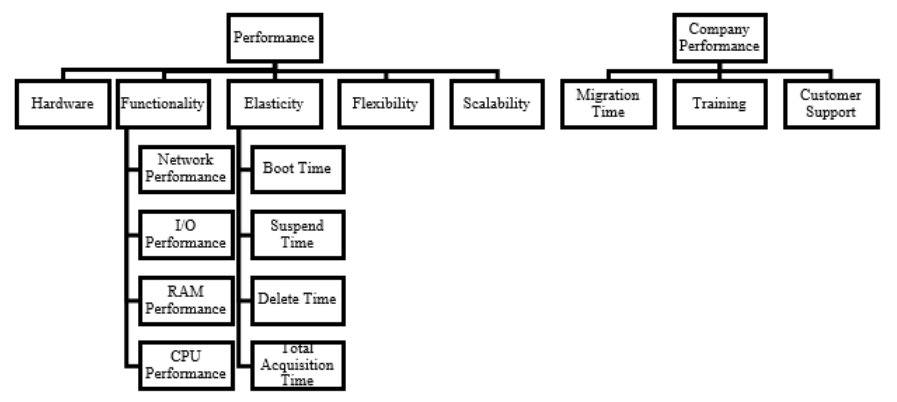

Figure 8 QoS Attributes for Performance and Company Performance

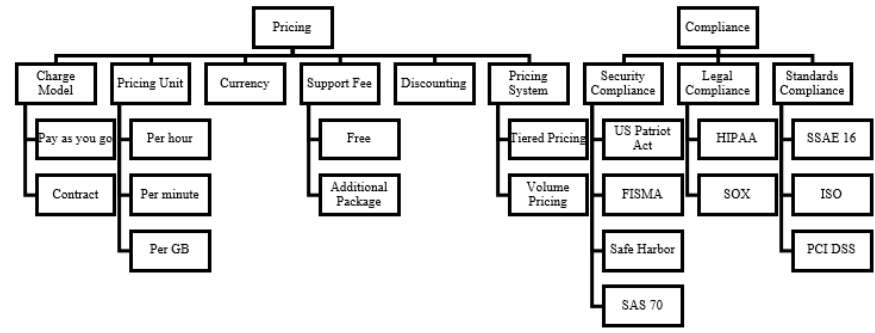

Figure 9 QoS Attributes for Pricing and Compliance

Now that the QoS attributes in the proposed model have been structured, the comparison between the three existing tools and the proposed model is detailed in the Table 3.

TABLE 2 QoS ATtRibutes COMPARISON BetweEn ToOls AND PROPOSED MODEL

\begin{tabular}{|c|c|c|c|c|c|}
\hline Criteria & $\begin{array}{c}\text { Intel } \\
\text { Cloud } \\
\text { Finder- } \\
\text { Quick } \\
\text { Search }\end{array}$ & $\begin{array}{c}\text { Intel Cloud } \\
\text { Finder - } \\
\text { Detailed } \\
\text { Search }\end{array}$ & $\begin{array}{c}\text { Rank } \\
\text { Cloudz }\end{array}$ & $\begin{array}{c}\text { Cloudor } \\
\text { ado }\end{array}$ & $\begin{array}{c}\text { Proposed } \\
\text { Model }\end{array}$ \\
\hline Structure & $\begin{array}{l}\text { Non- } \\
\text { hierarchy }\end{array}$ & Hierarchy & $\begin{array}{l}\text { Non- } \\
\text { hierarchy }\end{array}$ & Hierarchy & Hierarchy \\
\hline \# of Level & 1 & 2 & 1 & 2 & 3 \\
\hline $\begin{array}{l}\text { Flexibility in } \\
\text { hierarchy }\end{array}$ & None & None & None & None & Yes \\
\hline User Input & $\begin{array}{l}\text { Option } \\
\text { selection }\end{array}$ & Importance & Importance & $\begin{array}{l}\text { Option } \\
\text { selection }\end{array}$ & $\begin{array}{l}\text { Importanc } \\
\text { e and } \\
\text { option } \\
\text { selection }\end{array}$ \\
\hline Description & Partial & Available & None & Available & Available \\
\hline
\end{tabular}

The proposed model for representing QoS attributes combines the best value for each criteria. In it, QoS attributes are placed in a hierarchical structure in order to make it easier for user to see a clear categorization of the myriads of attributes There is flexibility in the hierarchy where in the cloud service selection tool user has the choice to see the QoS attributes in different depth, for instance the choice to see a 2level hierarchy or a 3-level hierarchy. The type of input will be different depending on the hierarchy level that user chooses. At the top level user needs to determine the importance of QoS attributes, while at the lowest level in the hierarchy user needs need to select from a set of options. Lastly brief description for all attributes will be provided to help users have some understanding.

\section{CONCLUSION}

In this paper we have compared and analysed the cloud QoS attributes representation in three existing cloud service selection tools. These tools have different ways of representing QoS attributes. We have compared the QoS attributes representation based on structure, number of level, flexibility in hierarchy, type of user input, and description of QoS attributes. Based on these five criteria, each tool represents the QoS attributes in various ways that are different from one another but none of these tools represent QoS attributes in the best way possible.

We have also proposed a new model to succinctly represent QoS attributes which cloud consumers can easily understand. The proposed QoS attributes representation model combine the best value for each of the five aforementioned criteria. In addition, in order to cover broad aspects of cloud computing the proposed model also makes sure that the QoS attributes included in the model covers technical, organizational, economic and political areas of cloud computing. The resulting QoS attributes representation is ready to be utilized for multi-criteria decision making (MCDM)-based cloud selection process as it conforms to the way MCDM technique breaks down a problem. In the future, work can be done in applying the proposed model to a cloud service selection tool that is based on an MCDM technique. By applying the attributes representation model, we will be able to see how user interacts with the QoS attributes representation proposed in this study as well as to gauge the simplicity and effectiveness of the proposed model.

\section{REFERENCES}

[1] P. Mell and T. Grance, "The NIST definition of cloud computing", 2011.

[2] "Forbes Welcome", Forbes.com, 2017. [Online]. Available: https://www.forbes.com/sites/louiscolumbus/2017/04/29/roundup-ofcloud-computing-forecasts-2017/\#256ca7ca31e8. [Accessed: 08- Dec2017].

[3] M. Salama, A. Shawish, A. Zeid and M. Kouta, "Integrated QoS UtilityBased Model for Cloud Computing Service Provider Selection", 2012 IEEE 36th Annual Computer Software and Applications Conference Workshops, 2012.

[4] CSMIC, "Service Measurement Index Framework Version 2.1", 2014. [Online]. http://csmic.org/downloads/SMI_Overview_TwoPointOne.pdf/. [Accessed: 08- Dec- 2017]. 
[5] S. Garg, S. Versteeg and R. Buyya, "SMICloud: A Framework for Comparing and Ranking Cloud Services", 2011 Fourth IEEE International Conference on Utility and Cloud Computing, 2011.

[6] S. Garg, S. Versteeg and R. Buyya, "A framework for ranking of cloud computing services", Future Generation Computer Systems, vol. 29, no. 4, pp. 1012-1023, 2013.

[7] G. Baranwal and D. Vidyarthi, "A framework for selection of best cloud service provider using ranked voting method", 2014 IEEE International Advance Computing Conference (IACC), 2014.

[8] Janssen, M., Joha, A., "Challenges for Adopting Cloud-based Software as a Service (SaaS) in the Public Sector," ECIS 2011 Proceedings, 2011.

[9] L. Baldwin, Z. Irani and P. Love, "Outsourcing information systems: drawing lessons from a banking case study", European Journal of Information Systems, vol. 10, no. 1, pp. 15-24, 2001.

[10] "Guidelines for Quality Management in Soil and Plant Laboratories", Fao.org, 2017. [Online]. Available: http://www.fao.org/docrep/w7295e/w7295e00.htm. [Accessed: 16- Dec2017].

[11] "ISO 9000:2015 Quality management systems -- Fundamentals and vocabulary", ISO, Geneva, 2015.

[12] "Intel ${ }^{\circ}$ Cloud Finder - Best Cloud Service and Solution providers", Intelcloudfinder.com, 2017. [Online]. Available: http://www.intelcloudfinder.com. [Accessed: 30- Aug- 2015].

[13] "Compare Clouds | Rank Cloud Services | Best IaaS | RightCloudz", Rightcloudz.com, 2017. [Online]. Available: https://rightcloudz.com/. [Accessed: 30- Aug- 2015].

[14] "Cloud Computing Comparison Engine", Cloudorado - Cloud Computing Comparison Engine, 2017. [Online]. Available: https://www.cloudorado.com/. [Accessed: 08- Dec- 2017].

[15] A. Polyviou, N. Pouloudi and S. Rizou, "Which Factors Affect Software-as-a-Service Selection the Most? A Study from the Customer's and the Vendor's Perspective", 2014 47th Hawaii International Conference on System Sciences, 2014. 